\title{
Wireless networks for medical applications
}

\author{
Cornelius A. D. Pahalson \\ Department of Science, Plateau State Polytechnic, Barkin Ladi, Nigeria
}

\begin{abstract}
With recent developments in the wireless networks field, new and innovative medical applications based on this technology are being developed in the research as well as commercial sectors. This trend has just started and the paper predict wireless networks are going to become an integral part of medical solutions due to its benefits in cutting down healthcare costs and increasing accessibility for patients as well as increasing the efficiency of the healthcare professionals. Same papers give some background on applications of wireless networks in the medical field and discuss the issue and challenges. It also tried to identify some of the standards in use. Another contribution due to this paper is the identification of innovative medical applications of wireless networks developed or currently being developed in the research and business sectors and also talk about the future trends in this field.
\end{abstract}

Keywords: Medical Applications; Wireless Networks; Sensor Networks; Wireless Applications; Wireless Homecare; Smarthomes; WBAN; Patient Management; Codeblue; Wireless Medical Solutions

\section{INTRODUCTION}

Wireless networks technology has been consistently achieving with time and increasingly finding its way into all aspects of our daily lives. Medical application is a field where Wireless networking can be promoting future. In the healthcare field, access and cost saving are two of the important issues. Wireless Technologies can contribute towards solving these issues.

The healthcare system is continuously getting more complex around the world but especially in the United State, 98,000 patients die each year as a result of preventable medical errors (Vawdrey, Hall, Knutson, Archibald, 2003). These are errors that could have been avoided. The Physicians and Clinicians most times provide patient care without knowing history of prescriptions and medical procedures, resulting both wasteful duplication and sketchy clinical decisions that do not take into account critical data related patient health. Wireless technology offers tools that can help such situations. Wireless Network reveals the caregivers real-time access to accurate patient data, clinical histories, treatments, medications, tests, lab results, insurance information and etc. 
With the wireless applications, healthcare providers such as hospitals, insurance agencies and the government are becoming interested in investing in this area. Cost saving is one of the main factors because medical errors by doctors brings in law suits. Patient and hospital management can be very expensive too.

\section{WIRELESS NETWORKS FOR MEDICAL APPLICATIONS}

Due to advances in the wireless networks field, new and innovative applications are being thought of in medical as well as healthcare field. In the medical field applications ranging from equipment management to patient management are being developed. Efficiency among hospital staff is increased by using some of this newly available applications and tools. In the healthcare field, issues such as long-term patient care support for elderly people and smart homes are being discussed in the realm of wireless networks. There is also research being done on creating teletrauma systems (Chu and Ganz, 2004) using the wireless channel. This will potentially allow trauma specialist to be virtually on patients bed sides while they are being moved to the trauma centre. In the near future homes can be designed that take care of patients or people with disabilities without the presence of a healthcare provider. A patient who is located remotely can be cared for remotely by communicating his/her status in real-time to caregivers. Another issue that concerns the healthcare field is the very large number of expensive medical devices that are incompatible with each other. Tedious routines are involved in translating results from one machine to another. With Wireless Technology this compatibility issue can be reduced.

Another hot issue in the Wireless networks field is implantable devices. These devices can be implanted on normal day to day wearable (Malan, Fulford-Jones, Welsh, and Moulton, 2004). Wireless sensors implanted inside patient's body have their own significant benefits (Timmons and Scanlon, 2004) and (Scanlon, 2003). Patients can wear sensors that monitor vital signs and report them in real-time to their doctors. This helps towards the issue of access because now the patients don't need to be around the hospital all the time. This improves access and quality of healthcare for patients and saves money for care providers.

\section{1. Benefits}

Whenever a new technology or tool is introduced to public use, the first question is, how will it help me or what can it do for me? Here are some benefits from applications of wireless networks in the healthcare field.

We hear in the news every day that there is a shortage of specialized doctors or nurse around the country. One of the benefits of these applications is that they increase doctors' and nurse' efficiency. Now they can care for more patients than before. Their work is also improved and made easier by having access to accurate data on patients in real-time.

For patients whose health is on the line the benefits are even greater. They have increased access to specialized doctors. They don't have to stick around the hospital any longer. This ease in mobility allows them to do their own work while still under the doctor's care. Safety is another issue that is helped here because the rate of mistakes can significantly be decreased. Also, patients can be picky when making changes in their daily lives when signing up for a treatment. With wireless Technologies, their healthcare can be less intrusive, for example in this case of wearable sensor (Malan, et al, 2004). They don't have to show up to the hospital for a blood pressure check. It can be done while they are working through wearing wireless sensors that transmit this information in their doctors. 
As mentioned earlier, negligent mistakes by doctors and nurses costs hospitals, insurance companies and the government a large sum of money each year. These costs can be reduced by reducing the number of mistakes. Efficient and secure data handling and resource management is another area where wireless networks can help. Deploying huge machines around the hospital can be very expensive and time consuming. With Wireless Technology interfaces can be designed such that access to the machines can be provided from anywhere in hospital. This allows for rapid and flexible deployment. By increasing the doctors' and nurses' efficiency, hospitals can provide care for more patients and increase their profits.

\section{2. Wireless Networks Technologies}

Due to accessibility and mobility requirement, wireless is the preferred medium in medical applications. Due to limited mobility, most of the expensive and large machines that have around for a long time are not being used to their fullest. Wireless technologies are being developed to give new interfaces to these machines and make them interact with any new machines and procedures required in medical field. Some recent and past wireless network based technologies in medical application includes.

\section{a. Wireless Body Area Network (WBAN)}

Recent advances in sensors lower powered integrated circuits and wireless networks have brought ideas in developing low powered physiological sensor platforms that can be integrated in Body Area Networks (BANS). These sensor networks have extremely low power requirements which makes them fit for integrating them in day to day wearable (Malan,, etal, 2004). Medical application, unobtrusive devices are attached to patients' bodies to collect vital health information such as ECG, Blood pressure etc. (Jovanov, Milenkovic, Otto, Groen, Johnson, Warren, Taibi, 2005). When used inside hospitals BANs are used to monitor patients in critical conditions. Outside the hospital, i.e homecare, patients 'Vital signs can be collected and transmitted over the internet tou their doctors and nurses in real-time. Besides medical applications, BANs are also finding use in fashion and defense fields.

\section{b. Radio Frequency Identifications (RFID)}

Radio Frequency Identification ( RFID) technology is a topics these days both in terms of its potential benefits and misuses. RFID tag are used in hospitals to keep track of equipment. They can also be planted on patients as well as doctors to know at a time where they are. (1) RFID are extremely low powered radio devices that don't need any battery power and thus have potential uses in storage areas. (2) Other potential used are in monitoring hospital supply stocks. Hospitals can manage their resource properly and know in real-time the status of their supplies.

\section{c. Wireless Personal Area Network (WPAN)}

WPANS (Chevrollier, and Golmie, 2005), (Golmie, Cypher, Rebala, 2005) and (Golmie Cypher, and Rebala, 2004) using 802.15.4 or Bluetooth have potential uses in the medical fields. These are short range networks that can be deployed for example, within a patient's room. Nurses are able to monitor patients in real-time without having to visit them frequently. This saves them time and gives them the opportunity to take care of more patients. Other uses of WPANs are in interfacing multiple expensive and large devices within the hospital. Data from one device can be forwarded directly from one machine to another 
without doctors wasting time by transferring this information by hand. Bluetooth is also a good technology for short range communication, for example, in home healthcare.

\section{d. Sensor Networks}

sensor Networks technologies such as Zigbee (Chevrollier, Golmie, 2005) are being combined with WBANs (Jovanov, et al, 2005) to form smaller scale networks that can be placed on human clothing (or other objects) and provide unobtrusive access to their health information. Sensor Networks are also increasingly being used in natural sciences for example in monitoring wild life or other natural phenomenon. Due to lower power requirements they can be deployed for a long period of time. Due to limited range, they are deployed in large numbers and thus form a distributed network covering a large portion of space. A good example of an application of sensor networks in medical field is the CodeBlue project (Malan, et al, 2004) being developed at Harvard. Other experimental applications include forest fire detection and path tracking using adhoc sensor networks (Fok, Roman, Chenyang , 2004). Since sensors networks device are very cheap, they can be deployed anywhere in large numbers. Some of the wireless sensor networks based devices are very sophisticated. They operate on their own operating system called the TinyOS. Therefore they can be programmed over the air, making their management very easy.

\section{e. GPRS/UMTS}

GPRS and UMTS wireless technologies have also found their uses in the area of medical applications. An application called MobiHealth, which, had been designed by using BANs with GPRS/UMTS for internet connectivity. i.e to create a generic platform for home healthcare using BAN-based sensors and wireless telephony technology. They are using GPRS/UMTS wireless communication technology for transferring data. Some of its capabilities include measurement and transmission of vital signs and other biosignals.

\section{f. Wireless LAN (802.11)}

Most hospitals, universities and corporate offices these days provide wireless LAN access. Some benefits include untethered access to the internet. Hospitals can use the wireless LAN channels to transfer patient data around the hospital. Communication between medical devices is also made possible using this wireless channel.

\section{3. Standards}

Although the area of wireless networks for medical applications is largely without standards and recently there are increasing demands for creating strict standards, especially for pacemakers, which are being used by a large number of heart patients across America.

A. IFEE Standards Medical Device Communications/Health Information Standards subscription

- ISO/IFEE Health informatics- Point-of-care medical device communication

- IFEE Standard for Medical Device Communications-Transport Profile

B. Mobile Health Care Alliance

$\circ \quad$ Setting Standards for mobile health information systems 
C. Medical Implant Communications Service in the $402-405 \mathrm{MHz}$ band

o High-speed, ultra power, non-voice transmissions to and from implanted medical devices.

\section{4. Challenges and Issues}

With new technologies, new applications and solutions are finding their way into different fields, but challenges and issues that didn't either exist before or are worsened. The medical field has the strictest quality and assurance requirements. Because patients' sensitive information and their well being are at stake. New challenges arise on how to tackle these issues. Some of the challenges faced when deploying Wireless network-based solutions include engineering issues, social issues and patients wellbeing issues. An implementation related challenge is interoperability among various devices. For example wireless devices are developed by different companies which follow different standards and in some cases no standards. Therefore making sure that all these devices work together and work reliability is a challenge. For example, different wireless medical devices working at different frequencies. One cannot assume that a device that works once will always work. In the medical field, strict guarantees are needed because a patients well being may depend on it.

In applications where there are real-time requirements, it can be challenging when guaranteeing real-time service. Wireless devices can behave differently at different times and locations due to natural and unnatural issues. Power requirements and power availability maybe in different situations. Some devices may fail during operation. Guaranteeing a seamless service during bandwidth jitters and handling total disconnection can be challenging As mentioned earlier, reliability is one of the most important issues in medical applications. Hospitals will not be willing to use a wireless device or application that cannot guarantee reliability due to the fear of lawsuits and other costs in the case of failure.

For patients, one of the most important issues is how their daily life can be affected by using these new applications. Thus designing applications that can be useful while unobtrusive is another challenge for wireless networks based application/solution developers. Since most wireless networks based devices are battery operated, one of the major challenges for developers is the issue of power limitations. Sometimes they have to guarantee that the device will work for a year or two without changing the battery (Culpepper, Dung, and Moh, 2004). This could include devices such as heart pacemakers (Culpepper, et al 2004). The developers have to design better scheduling algorithms and power management schemes to deal with these power issues. Also we know that wireless channels are slower than wired. Developing applications/ devices that can deal with traffic congestion and other performance issues, is a major challenge.

Some main issues that arise due to the use of wireless networks devices include security, privacy and the learning curve fir new technologies. Ensuring patients information security can be a major issue when deploying these applications. Privacy of user data over wireless channels can be another major issue. Wireless networks based medical devices can be very limited in terms of power availability and processing strength. Thus ensuring privacy without using complex encryption algorithms can be a big issue for developers of medical devices. With the new technologies taking hold in our daily lives, new users can find it challenging to use these new devices to the fullest. Thus it can be an issue and a challenge for developers to create some of the best solutions without forcing the users to make unnecessary of effort just to learn how to use them. 


\section{5. Future Trends}

Although wireless technology in the field of medical applications is still relatively new, commercial products are being developed by several companies to solve wide ranging problems. In some cases these new applications are design purely social health benefits i.e. reducing interference to daily life when dealing with long term patient care. The trends in wireless networks use in medical applications of what's to come include:

a. Patient Homecare is a new area in the use of wireless networks for medical applications (Fishky and Weng, 2003). Homecare has a central role in the emerging medical applications of technology ( Stankovic, Cao, Doan, Fang, He, Kiren, Lin, Son, Stoleru, 2005) Remote monitoring of patients and the elderly inside their homes will significantly reduce costs for caregivers and also provide a non-intrusive lifestyle for its users (Fishky et al, 2003).

Some applications include:

- Medicine intake tracking

- Remote ECG collecting and monitoring

- Remote blood pressure and weight monitoring

- Wearable sensors as part of a BAN to monitor vital signs and other biosignals.

- Monitoring and control of remote devices

Some benefits of using wireless networks based technologies in Homecare include:

- Non-invasive monitoring

- Cost saving and efficient method of care

- Ease of communication between care givers and patients

- As we are talking about trends in the wireless networks for medical

Applications field, some potentially useful applications in the homecare sector include:

- In-home assistance

- Smart nursing homes

- Clinical trial and research augmentation

- Control of home appliances

- Emergency Communication

- Medical data lookup

- Memory enhancement

b. Context-Sensitive Medicine; By using Active RFID-tags, management of resources becomes more intelligent. For example machines needing servicing can automatically inform the technician. Patient care can be scheduled and reminders will be sent out exactly when something needs to be done. The location of equipment, doctors, patient etc. inside a hospital can be tracked in real-time. This allows the patients to move around freely. It also allows doctors and nurses to tend to more patients. Besides potential uses in the medical field, context-Sensitive technology have military uses as well (Keeping track of military equipment is an important issue). Wireless technology employed:

- Active-RFID

○ Send signals indication location etc.

- Radiance Indoor Positioning Solution (IPS) 
- WLAN

Some potential applications of Context-Sensitive Medicine:

- Workflow

- Equipment Tracking

- Patient Location

- Patient Information Retrieval During Emergencies (Vajirkar, Singh, Lee, 2003)

- Single-Use Location Tag

The following figure describes a basic architecture based on the Context-Sensitive Medicine platform. An Active RFID-tag is attached an object (equipment, patient, doctors etc.). The tag continuously sends out updates over the wireless channel to a wireless receiver which is in communication with a central server in the hospital. The server can take action or forward that information to other mobile devices or email accounts of people in charge.

c. IRevive: IRevive is a commercial project that is based on the CodeBlue research project from Harvard. It provides mobile device synchronization with a central database server. These mobile devices can capture vital signs information in real-time and store using VitalDust technology (CodeBlue) (Shnayder, Chen, Lorinez, Thaddeus Fulford-Jones, and welsh, 2005) or send them to a central server..

\section{CONCLUSIONS}

Wireless networks for medical Applications are becoming important topics in the industry. With its potential uses in the medical and healthcare fields, wireless networks have an important contribution in improving lives of patients. There are large commercial benefits in the area of reducing costs and improving equipment and patient management.

The paper, highlighted the benefits of using wireless networks for medical applications, and can as potentially reduce costs for hospitals, government and insurance companies. With wireless networks-based medical technologies, applications can be designed to be less intrusive in patients' lives.

All applications based on scientific methods have a development lifecycle. This is normally starting out with a research work and moving unto commercialization's in the future. We talked about current and past research projects. We also brought in some commercial applications that are currently available in the market. Some of these products are very innovative and have chance $s$ of succeeding. With all new technologies, there are chances of failure and success.

\section{References}

[1] Culpepper B. J., Dung L., Moh (2004). "Design and Analysis of Hybrid Indirect Transmissions (HIT) for Data Gathering in Wireless Micro Sensor Networks, "ACM Mobile Computing and Communications Review (MC2R).

[2] Chu Y., Ganz A., IEEE Transactions on information Technology in Biomedicine 8 (2004) 450-462. 
[3] Chevrollie N., Golmie N. (2005). "On the use of wireless Network Technologies in Healthcare Environments" white paper-U.S Department of Commerce.

[4] Chevrollier N., Montavont N., Golmie O. (2005). "Handovers and interference mitigation in healthcare environment", to appear in the proceedings of the IEEE Military Communications Conference (MILCOM 2005).

[5] Chevrollier N., Golmie N. (2005) "On the use of Wireless Network Technologies in Healthcare Environments," proceeding of the fifth IEEE workshop on applications and Service in wireless networks (ASWN2005), pp. 147-152.

[6] Fok C. L., Roman G. C., Chenyang Lu (2004). "Efficient Network Exploration and fire Detection using Mobie Agents in a wireless Sensor Network" - (MURI ONR 3-years review, Baltimore, MD.

[7] Fulford-Jones T. R. F., Welsh G. Y. (2004). “A portable, low-Power, wireless two-lead EKG system.” In proc. $26^{\text {th }}$ IEEE EMBS Annual International Conference, San Francisco.

[8] Fishky K., Wang M. (2003). “A flexible, Low-Overhead Ubiquitous System for Medication Monitoring”, Inter. Research Technical Report IRS-TR-03-011.

[9] Golmie N., Cypher D., Rebala O., Computer Communications 28 (2005) 1255-1275.

[10] Golmie N., Cyphe D., Rebala O. (2004). "performance evaluation of low rate WPANs for sensor and medical applications." In Military Communications Conference (MILCOM 2005).

[11] Jovanov E., Milenovic A., Otto C., Groen P. D., Johnson B., Warren S., Taibi G. (2005). "A WBAN System for Ambulatory Monitoring of physical Activity and Health Status: Application and Challence," proceeding of the $27^{\text {th }}$ Annual International Conference of the IEEE Engineering in Medicine and Biology Society (EMBS), Shanghai, China.

[12] Jovanov E., Milenovic A., Otto C., Groen P. D. (2005). “A wireless body area network of intelligent motion sensor for computer assisted physical rehabilitation", Journal of Neuron Engineering and Rehabilitation.

[13] Malan D., Fulford-Jone T., Welsh M., Moulton S. Codeblue (2004). An ad hoc sensor network infrastructure for emergency medical care." In proceeding of the international workshop on wearable and implantable Body Sensor Networks.

[14] Stankovic J. A. et al. (2005). "Wireless Sensor Networks for In-Home Healthcare: Potential and Challenges", in High Confidence Medical Device Software and Systems (HCMDSS) Workshop, Philadelphia

[14] Shnayder V., Chen B-R. Lorincz K, Fulford-Jones T. R. F., Welsh M .(2005). "Sensor Networks for Medical Care”, Harvard University Technical Report TR-08-05.

[15] Scanlon W. G. (2003). "Analysis of tissue-coupled antennas for UHF intra-body communications," $12^{\text {th }}$ IEEE Intl. Conf. Antennas and propagation (IEEE Conf. publ. No. 491), Vol. 2, pp. 747-750.

[16] Timmons and Scalon W. (2004). "Analysis of the performance of IEEE 802.15.4 for medical sensor body area networking," IEEE Sensor and Ad Hoc Communication and Networks Conference (SECON). 
[17] Vawdrey D. K., Hall E. S., Knutson C. D., Archibald J. K. (2003). “A Self-Adapting Healthcare Information Infrastructure Using Mobile Computing Device”, Enterprise Networking and Computing in Healthcare Industry, 2003. Proceedings. $5^{\text {th }}$ International Workshop page(s): 91-97.

[18] Vajirkar P., Singh S., Lee Y. (2003). "Context-Aware Data Mining Framework for Wireless Medical Application”, Page 381-391. 